\title{
Norois
}

Environnement, aménagement, société

\section{Le logement social dans un pays rural sous influence urbaine : une forme hybride du périurbain}

Council housing in a rural area under urban influence: a suburban hybrid form

Yannick Sencébé

\section{OpenEdition}

Journals

Édition électronique

URL : http://journals.openedition.org/norois/1226

DOI : $10.4000 /$ norois. 1226

ISBN : 978-2-7535-1553-6

ISSN : $1760-8546$

Éditeur

Presses universitaires de Rennes

Édition imprimée

Date de publication : 1 décembre 2007

Pagination : 11-22

ISBN : 978-2-7535-0616-9

ISSN : 0029-182X

\section{Référence électronique}

Yannick Sencébé, «Le logement social dans un pays rural sous influence urbaine : une forme hybride du périurbain », Norois [En ligne], 205 | 2007/4, mis en ligne le 01 décembre 2009, consulté le 19 avril 2019. URL : http://journals.openedition.org/norois/1226 ; DOI : 10.4000/norois. 1226 


\title{
LE LOGEMENT SOCIAL DANS UN PAYS RURAL SOUS INFLUENCE URBAINE : UNE FORME HYBRIDE DU PÉRIURBAIN
}

\author{
YANNICK SEnCÉbÉ \\ CESAER - UMR 1041 INRA \\ (École nationale d'enseignement supérieur agronomique de Dijon), \\ 26 boulevard Docteur-Petitjean, BP 87999 - 21079 Dijon \\ yannick.sencebe@enesad.inra.fr
}

\begin{abstract}
RÉSUMÉ
Cet article présente les résultats d'une recherche exploratoire sur le logement social en milieu rural. Le propos vise à situer la nature et les acteurs en jeu dans l'implantation de ce type d'habitat en rapport avec l'influence urbaine qui s'exerce sur les communes rurales. Deux types d'enquête alimentent la réflexion. Une enquête quantitative montre l'importance du logement dans une politique de développement rural menée par la région Bourgogne. Elle resitue également la manière dont les élus ruraux s'approprient l'enjeu du logement locatif selon leur attractivité résidentielle vis-à-vis de la ville. Une enquête qualitative menée sur un pays rural de l'Yonne permet ensuite d'entrer plus précisément dans les relations entre acteurs. Tour à tour sont évoquées l'histoire politique locale du pays qui a favorisé la concurrence des élus dans la quête de nouveaux habitants, la nature complexe de cette forme d'habitat qui constitue à la fois un non-lieu et un lieu en devenir dans l'espace d'une commune sous influence d'Auxerre, et enfin la transaction sociale entre les élus ruraux et le bailleur social urbain d'Auxerre.
\end{abstract}

Mots CLÉ : Espace rural-Logement social-Périurbanisation-Sociologie.

\section{ABSTRACT \\ Council housing in a rural area under urban influence: a suburban hybrid form}

This paper outlines conclusions of a research in sociology on the council housing development in a rural area under urban influence. The analysis combines different levels of the phenomenon: the political history which favoured concurrence between councillors to attract new inhabitants, the complex nature of this settlement that hardly fit into the village area, and lastly the social dealing between councillors and urban social lessor.

KEY WORDS : Council housing - Rural area - Sociology - Suburban.

Cet article aborde la question de la place du logement locatif social en milieu rural. Depuis une date récente, la gestion du logement social est restée sous l'influence des politiques d'urbanisme conçues pour et par le monde urbain (Delannoy, 2005). Cependant, certaines évolutions permettent de penser que le logement social en milieu rural est un phénomène non négligeable 
et potentiellement amené à s'étendre. Des études éparses, menées par divers organismes (Fédération Nationale Habitat et Développement, Mairie Conseil, conseils économiques et sociaux de différentes régions) alimentent un début de réflexion en la matière. La rareté de l'offre locative en milieu rural (27\% du parc des résidences principales pour le locatif et $7 \%$ pour le locatif HLM, d'après Le Marec, 2004) ne doit pas occulter la demande croissante de logements qui s'y fait sentir à la faveur de plusieurs phénomènes aujourd'hui relativement bien connus (pression immobilière urbaine, augmentation du nombre de ménages, attractivité du rural) qui tracent les contours des « campagnes résidentielles » (Perrier-Cornet, 2002). En outre, l'espace rural, caractérisé par une relative disponibilité foncière, peut tirer parti de la préférence des ménages pour l'habitat plus individuel. Ainsi, un tiers des logements locatifs récents construits entre 1998 et 2001 sont des maisons, dans le secteur libre comme dans le secteur social (Minodier, 2004).

Au-delà de la ville à trois vitesses composant déjà une hiérarchie socio-spatiale autour des noyaux citadins (Donzelot, 2004), l'espace rural, sorte de " périurbain lointain », serait-il un sous-produit de la crise immobilière, rejetant toujours plus loin en périphérie des villes les ménages les plus modestes (Vanoni, 2007)?

La perspective suivie ici cherche plutôt à souligner la nature complexe de l'implantation de logements sociaux en milieu rural. Ainsi, les analyses réalisées tendent à montrer qu'au travers du logement social, il ne s'agit pas seulement de «loger» les pauvres mais aussi d'attirer et d'accueillir de nouveaux ménages dans une perspective de revitalisation ou de développement local. Les maires ont acquis depuis la décentralisation des compétences en matière d'urbanisme et le logement constitue un levier important de leur action. Ces stratégies communales doivent être référées à la diversité de situations vis-à-vis de l'influence urbaine. Certaines communes, en effet, profitent d'une attractivité résidentielle du fait de leur accessibilité aux pôles urbains tandis que d'autres se trouvent à l'écart de cette influence.

Nous avons fait le choix de traiter de la question du logement social en nous référant à différentes échelles : une région, un département, un pays et une commune. Si ces niveaux s'emboîtent géographiquement, nous verrons que la perception et le vécu des acteurs situés à chacun d'entre eux diffèrent considérablement. Afin de situer globalement la place et les enjeux du logement locatif et social en milieu rural, nous présentons tout d'abord les résultats d'une enquête menée à l'échelle de la Bourgogne, centrée sur l'analyse d'un dispositif régional d'aide au développement du locatif en milieu rural. Nous nous appuyons ensuite sur une étude menée à l'échelle d'un pays rural du département de l'Yonne (Puisaye-Forterre) dans le cadre d'une recherche collective initiée en 2005 sur le thème de la co-habitation en milieu rural ${ }^{1}$. Le volet présenté ici s'intègre donc à un dispositif plus large et intervient à la suite de différentes étapes. Des entretiens avec des personnes ressources et un cadrage sociodémographique de la zone nous ont permis de choisir une dizaine de communes dont les maires ont été interviewés sur différents sujets et notamment sur l'accueil de populations et les relations entre habitants. Différentes pistes ont ensuite été creusées comme relevant d'objets permettant de saisir la question de la co-habitation en milieu rural. La question du logement social, ici abordée, a été traitée à partir d'un recentrage sur une commune. Celle-ci, sans être représentative, présente l'intérêt de condenser un certain nombre d'évolutions significatives de l'urbanisation du rural. Cinq entretiens semi-directifs ont été menés avec le maire de cette commune, trois habitants du lotissement et la responsable de secteur de l'office HLM qui gère l'ensemble du parc social dans la zone. Ces entretiens ont été complétés par l'analyse de divers documents fournis par l'office HLM. Les résultats ici présentés sont donc à considérer comme relevant d'une démarche exploratoire et à conforter.

Pour saisir les différentes facettes du logement social dans ce secteur, nous ferons un détour par la genèse politique du pays qui explique la concurrence actuelle entre les maires dans la bataille

1. Cette recherche associe outre l'auteur du présent article, P. Alphandéry (Inra-Mona Ivry) et F. Pinton (Ladyss - Université Paris 10), dont l'investissement ancien sur le terrain de Puisaye a donné lieu à différentes publications (Alphandery, Pinton, 1998). 
pour le repeuplement de leur commune. Nous nous arrêterons un moment sur la nature même du lotissement social pavillonnaire en tant que forme hybride d'habitat, pour suivre enfin les méandres des transactions entre les élus locaux et le bailleur social urbain qui gère ces logements.

\section{Place et appropriation d'une politique régionale en faveur du logement locatif en milieu rural}

Dans cette région très rurale $(78 \%$ de la surface et les trois-quarts des communes sont situés dans l'espace à dominante rurale), le Conseil régional a mis en place, en 1999, un dispositif « cœurs de villages » que nous avons suivi à travers l'encadrement d'un travail universitaire (Zamboni, 2007). Structuré au départ en différents volets orientés vers des actions de revalorisation du bâti existant à destination des communes de moins de 3500 habitants (75\% des communes éligibles ont moins de 500 habitants), ce dispositif a été renforcé à partir de 2004 en ce qui concerne l'aide au logement social notamment. Cette politique, prioritaire en termes de crédits accordés (46 millions d'euro entre 1999 et 2006 alors que 10 millions ont été accordés durant la même période au titre des contrats d'agglomération), partait du constat d'un décalage entre la demande croissante de logement en milieu rural et l'existence d'un patrimoine non utilisé alors même que, dans une bonne partie du rural bourguignon, le risque de dévitalisation démographique et économique subsistait.

Une évaluation quantitative a été menée à partir du traitement des différents documents administratifs (dossiers de candidature, projets, actions réalisées, fonds attribués, impact...) et de deux enquêtes, la première par questionnaire auprès de 117 communes bénéficiaires de la mesure, et la seconde, par entretiens semi-directifs auprès de 18 maires dont l'échantillon a été constitué en tenant compte de leur répartition entre les 4 départements de la région, et entre l'espace périurbain (7 d'entre elles) et l'espace à dominante rurale (9 d'entre elles).

L'évaluation quantitative de cette politique, entre 1999 et 2006, montre que l'engagement des communes vis-à-vis du logement est important, et notamment parmi les petites communes. Fin 2006, parmi les 1982 communes éligibles, 770 communes avaient signé un accord-cadre. Concernant la place du logement, 1552 logements ont été réalisés ou étaient en voie d'achèvement, dont 645 logements sociaux (41\% des logements réalisés). Enfin, $85 \%$ des communes ayant réalisé leur investissement en matière d'habitat locatif et parmi elles, toutes celles qui comportent des logements sociaux, ont logé de nouveaux habitants. Ceci tend à valider l'hypothèse que le développement du logement locatif s'inscrit dans une perspective de développement local pour les élus ruraux. Cependant cette perspective générale semble prendre des formes différentes suivant la situation des communes vis-à-vis des pôles urbains.

L'enquête auprès des maires a permis en effet de mettre en évidence que pour les communes qui ne bénéficient pas de l'attractivité d'un pôle urbain, l'enjeu du logement est prioritaire et s'inscrit dans la perspective d'un maintien démographique. Il s'agit à travers la création de nouveaux logements d'installer de nouveaux habitants, de préférence avec enfants, et de préserver une vie sociale et la présence des services publics. Ainsi, parmi ces maires ruraux, l'argument de l'école reste le plus fort.

Au contraire, pour les communes attractives et proches d'un pôle urbain, le développement du logement locatif au sein de leur commune est à considérer dans une politique globale d'aménagement et de développement du village. "Cœurs de Villages » apparaît ici comme une étape pour augmenter l'attractivité de la commune en diversifiant par exemple l'offre de services déjà existante alors que pour les communes moins attractives, cette politique reste le seul moyen de préserver une vie sociale au village.

Entre la région et les communes, le département a vu ses compétences renforcées en matière de logement social (gestion du fonds de solidarité logement notamment). Dans l'Yonne, la gestion du logement social se fait par l'intermédiaire d'un office public situé à Auxerre mais dont le champ 
d'action s'étend de plus en plus en périphérie rurale. Penchons-nous à présent sur cette gestion urbaine du logement social en milieu rural pour tentez d'en comprendre les enjeux.

\section{Place et gestion du logement social en milieu rural : le cas de l'Yonne}

L'OPAC 89 est le seul bailleur social de l'Yonne. Créé par le Conseil général en 1950, sous le statut d'office public d'HLM du département, il a été transformé en Office Public et d'Aménagement et de Construction (OPAC) de l'Yonne en 1991. Depuis, il est géré par un conseil d'administration composé de 21 membres désignés par les différents représentants des pouvoirs publics et des élus. Il est épaulé par une commission d'appel d'offre et par trois commissions d'attribution des logements, organisées par secteurs géographiques, nord, sud, et centre, ce dernier associant Auxerre et la Puisaye-Forterre.

Sur les 8500 logements de son parc locatif, l'OPAC en gère 800 en Puisaye-Forterre (sur les 12802 résidences principales que compte le pays), dont nous traiterons ensuite plus précisément. 55 communes sur 62 sont couvertes par cette offre locative. Ce territoire proche d'Auxerre offre ainsi un débouché foncier non négligeable pour satisfaire la demande en logement individuel (type pavillon) qui va croissante parmi les ménages non solvables sur le marché classique et qui est très largement soutenue par les communes. Celles-ci se livrent à une véritable compétition pour la construction de logements sociaux, ce qui laisse à l'OPAC d'Auxerre le rôle d'arbitre. Les maires, soucieux de répondre aux demandes de leurs administrés mal logés (personnes âgées, jeunes couples) et d'assurer le développement de leur commune, vont ainsi défendre leurs dossiers devant une commission chargée des programmes de constructions et de réhabilitations immobilières. Le souci du logement social n'est pas toujours assorti d'une réflexion, chez les élus, sur l'insertion locale de leurs occupants et c'est l'OPAC qui édicte en la matière certains critères visant à limiter les «ghettos ruraux ». Le désengagement local de l'État, le desserrement urbain alimenté par la pression foncière et immobilière qui relègue les plus pauvres dans les périphéries rurales, la concurrence locale sur le parc privé avec les résidents secondaires favorisent ainsi une sorte de fuite en avant des élus locaux dans la construction de logements sociaux, à l'inverse de ce qui se passe dans les villes.

À partir d'entretiens auprès de la responsable du secteur Puisaye-Forterre (madame C.) et de données complémentaires, nous avons cherché à approfondir certaines de ces pistes et hypothèses. Deux points marquants ressortent : l'évolution des formes et de la demande de logements sociaux en milieu rural est semblable à ce qu'elle est en milieu urbain; la relation entre les élus ruraux et le bailleur social urbain relève d'un compromis où chacun, à partir de stratégies différentes, trouve un intérêt à développer le logement social en milieu rural.

\section{L'ÉVOLUTION DES FORMES ET DE LA DEMANDE DE LOGEMENTS SOCIAUX EN MILIEU RURAL : « COMME PARTOUT EN FranCE »}

Précisons d'emblée que la présence du logement social dans les communes rurales de l'Yonne est ancienne : les premières habitations gérées dans ce type de communes par l'OPAC 89 datent des années 1960. Madame C., dans sa description de l'évolution du parc de logement et de la demande, s'attache à montrer le parallèle avec la ville.

Dans les années 1960-1970, l’OPAC a commencé à construire dans les bourgs ruraux, des ensembles de plus de 50 logements, en collectifs « comme partout en France ». À partir des années 1975, l'OPAC s'est lancé dans les logements « modelec » (type de financement) avec des pavillons de plain-pied. Ces maisons, sans architecture particulière, n’ont pas spécialement bien vieilli, et font partie d'un programme de réhabilitation. Mais contrairement aux immeubles collectifs, ce sont des types d'habitat très prisés aujourd'hui. Ces logements représentent en outre pour l'OPAC un « produit » à forte rentabilité, sans aucune vacance. 
Cette évolution de la demande vers du logement individuel en milieu rural semble généralisée en France. Lors du colloque organisé par la Fédération nationale de l'Habitat Rural en mars 1987, le directeur de la Fédération des Associations Régionales d’Organismes HLM soulignait la même tendance : Ainsi les HLM sont présents sur l'ensemble du territoire, ils interviennent de plus en plus dans les bourgs ruraux et dans les petites villes sous la forme de l'acquisition amélioration en PLA (prêts locatifs aidés) mais aussi de constructions neuves, parfois en tout petits collectifs, le plus fréquemment en maison individuelle (Coulmin, 1988).

À partir de 1990, l'OPAC abandonne complètement le collectif pour investir sur « de petites cages de lotissements pavillonnaires ${ }^{2} »$. Cette expression, associant la référence aux cages d'immeubles collectifs et à l'habitat individuel, exprime assez bien le compromis trouvé entre la réponse à une demande plus exigeante (en habitat individuel avec petit terrain) et le maintien d'un prix de construction et de location accessible.

Cette orientation vers le logement individuel répond, selon Madame C., à l'évolution de la demande qui est en milieu rural à l'image de ce qui ce passe en milieu urbain : "C'est fini depuis 20 ans, le collectif pur en milieu rural. C'est partout pareil en France. Les gens ne sont plus prêts à aller dans un habitat collectif, type barre, parce que ce qu'ils souhaitent c'est le petit pavillon avec le petit jardin et être tranquilles chez eux. »

La construction de logement collectif, si elle existe encore, prend des formes totalement renouvelées qui empruntent au modèle de résidentialisation des grands ensembles ${ }^{3}$ (Dunoyer de Segonzac et al., 2004).

Quant à la gestion du bâti collectif existant, l'OPAC, après avoir entamé comme partout ailleurs des programmes de rénovation, en vient à prospecter du côté des « grands remèdes » employés dans les quartiers urbains sensibles : une étude sur le devenir de ces logements collectifs qui ont mal vieilli a été lancée et un programme de démolition de certains ensembles collectifs à fort taux de vacances est envisagé pour l'ensemble des communes. Cette stratégie est urgente selon l'OPAC qui craint l'effet tache d'huile en matière de vacance des logements. La stigmatisation que vivent certains habitants de quartiers difficiles n'est pas réservée au milieu urbain : en milieu rural, où la forte interconnaissance et la faible densité accentuent la visibilité sociale, l'effet quartier existe aussi et explique le fort taux de vacance des « ensembles collectifs » et l'ardeur de l'OPAC à réhabiliter jusqu'aux noms de rue : "Je vais vous donner l'exemple de Bléneau, on avait deux grandes barres et deux plus petites qui avaient été construites dans les années 60. On vient de refaire un coup de neuf dessus. Rien que pour vous dire, ça s'appelait la rue de la cité. C'était pas vendeur pour les louer. Donc ça a été rebaptisé, la rue des Grappes. On a aussi redessiné la place et c'est assez sympa. »

L'évolution de la demande, à laquelle cherche à répondre l'OPAC, est hétérogène. Trois grands groupes de populations semblent identifiables : les personnes âgées, nombreuses en milieu rural, qui cherchent à quitter des habitats individuels et vétustes pour s'installer dans des logements locatifs plus proches des services et des commerces; des ménages modestes enracinés localement et désirant rester dans leur commune ou à proximité de leur emploi et enfin des ménages d'actifs à Auxerre, prêts à faire de longs trajets quotidiens, pour accéder à un logement social individuel beaucoup plus long à obtenir en ville qu'à la campagne. Un partage du territoire de la PuisayeForterre s'effectue entre ces différents groupes selon une géométrie complexe. La périurbanisation de la Puisaye-Forterre, qu'alimentent les actifs urbains, suit un gradient qui ne s'inscrit pas dans une logique de distance kilométrique au pôle d'emploi urbain mais plutôt dans la logique aréolaire associant accessibilité à l'emploi urbain et accessibilité locale aux services et commerces.

"Aujourd'hui les personnes qui demandent Auxerre sont prêtes à faire trente kilomètres et Toucy (chef-lieu de canton) est dans le rayon, où tous les services sont présents en plus. Mais plus on s'enfonce

2. Les extraits suivants sont tirés de l'entretien avec madame C. réalisé à Saint-Fargeau dans les locaux de la maison du pays de Puisaye-Forterre, le 10 mars 2006.

3. Il s'agit notamment de normer les usages de chaque espace et d'en délimiter les parties privées et publiques, afin de rendre plus sûre leur fréquentation et de faciliter l'identification des habitants à la résidence, espace intermédiaire entre le logement et le quartier. 
dans la Puisaye rurale, plus la logique de demande va correspondre à des personnes qui ont un emploi ou des racines sur place. Plus la commune est petite, moins elle a de services, moins on a de demandes. Ceci dit, cela évolue, si elle a du logement individuel, les gens n'hésitent pas à s'y installer. »

Mais l'implantation de logements sociaux, et notamment de lotissements pavillonnaires, si elle suit une demande, ne serait pas possible sans l'accord et la demande expresse des élus locaux qui ont en charge depuis la décentralisation l'urbanisme de leur commune.

\section{GeSTiOn COMMUNALE DES ÉLUS ET GESTION LOCATIVE DE L'OPAC : UNE TRANSACTION PAS SEULEMENT IMMOBILIÈRE}

Transaction : le terme peut prêter à confusion lorsqu'il est question d'habitat. Il est utilisé ici dans le sens sociologique où l'entend J. Rémy (1998) et non dans le sens immobilier. La relation entre le bailleur social et les élus de Puisaye-Forterre peut se lire comme une situation dont la structuration est ouverte sur plusieurs réactions possibles. Les acteurs en jeu sont reliés par des exigences incontournables (développer le logement sur sa commune/développer son activité) mais partiellement incompatibles (prendre le risque d'investir en milieu rural; prendre le risque de voir un acteur extérieur s'immiscer dans la gestion de sa commune). Cette situation crée des interdépendances qui vont jouer un rôle socialisateur : chacun apprend à transiger et intègre une partie des intérêts de l'autre en faisant coïncider ses propres valeurs avec les orientations de l'autre.

Des deux côtés, les intérêts et les stratégies ne sont pas forcément congruents, mais ils convergent à l'issue d'une suite d'arrangements, d'engagements réciproques et de services mutuels. Autant d'échanges qui interdisent de trancher définitivement la question suivante : lequel des deux, au final, exerce sur l'autre un pouvoir, une domination? Chacun semble s'y retrouver, bien que l'on puisse émettre l'hypothèse que l'émiettement communal, évoqué plus haut, joue parfois en défaveur des élus.

Un lien fort existe entre l'OPAC et la Puisaye-Forterre, surtout depuis que le président du pays exerce également les fonctions de président du conseil d'administration de l'OPAC.

Ceci explique sans doute que la zone soit l'une de celles qui connaît le plus important développement de logements sociaux. Les différentes casquettes du président de pays et de l'OPAC, également conseiller général, ont sans doute facilité la signature des contrats d'objectif entre l'OPAC et les communes de Puisaye-Forterre. Il s'agit d'une convention que l'OPAC signe avec le Conseil général, les communes et l'État et qui engage le bailleur social à construire ou réhabiliter des logements, notamment dans des petites communes. Mais cet engagement laisse cependant parfois à l'OPAC le rôle d'arbitre quant à la localisation et à la répartition des logements. Lorsqu'une dynamique intercommunale existe, comme sur la communauté de communes de Bléneau, l’instruction des dossiers de demande se fait en collaboration entre le service « partenariat au développement » de l'OPAC et les élus. Mais en l'absence de concertation des élus locaux, c'est l'OPAC qui décide à travers une étude s'appuyant sur différents critères de rentabilité économique : l'adéquation entre la demande des élus et la demande en location, l'existence de patrimoine déjà géré par l'OPAC, et le taux de rotation des locataires dans les communes demandeuses.

Cette relation ambivalente de pouvoir entre les deux types d'interlocuteurs se retrouve dans la gestion des attributions des logements. La commission d'attribution réunit, une fois par mois, les six administrateurs de l'OPAC ainsi que les maires qui ont une voix délibérative prépondérante pour accepter ou refuser les demandes de logement dans leur commune. Néanmoins, c'est bien le bailleur qui convoque à Auxerre les maires des communes concernées. L'information à disposition des uns et des autres, autrement dit ce qui va étayer la défense ou le refus, n'est pas symétrique. Les dossiers de demande sont préparés par l'OPAC grâce à un formulaire très complet que doit remplir le demandeur. L'ensemble de ces dossiers va ensuite alimenter le fichier informatique des locataires de l'OPAC. Tout le parcours résidentiel de la personne y est pratiquement renseigné : outre la composition du ménage, le niveau et les sources de revenu, le lieu d'emploi, et même la possession d'animaux domestiques ou encore l'attente d'un enfant sont indiqués. Ce dossier est 
présenté à la commission qui est seule à trancher selon madame C. Cependant, des critères propres aux services instructeurs de l'office semblent également entrer en jeu : "Une des priorités, c'est l'objectivité du dossier sur la commune : quelqu'un qui habite à Sens et qui nous demande Mezilles [commune de Puisaye] on va se demander pourquoi? On regarde dans les demandes de logements si on peut pas avoir une logique de proximité qui peut passer par l'emploi, les racines, ou parce qu'il y a de la famille sur place, que la mamie peut garder les enfants."

Si le maire n'est pas sans ressources pour défendre et arbitrer les demandes de logement, grâce à la connaissance directe qu'il a des demandeurs, qui font souvent la démarche d'aller le rencontrer directement, cette connaissance ne concerne que ses administrés. Néanmoins le critère de proximité utilisé par les services de l'OPAC vient pallier cette dissymétrie de l'information et rejoint les préoccupations des élus : répondre à la demande de leurs administrés et électeurs, et maintenir la population sur place.

Enfin, la relation aux locataires n'incombe pas uniquement à l'OPAC, et c'est sans doute un avantage certain que ce bailleur social trouve en milieu rural. En effet, dans la gestion des impayés et des conflits de voisinage, l'OPAC fait largement appel aux maires. Une organisation spécifique pour le milieu rural a été mise en place : une commission itinérante composée d'administrateurs et de la responsable de secteur se rend dans les communes concernées en convoquant chez le maire, ce qui impressionne toujours en milieu rural, les locataires posant problèmes. Cette commission n'intervient cependant qu'en cas extrême. Dès les premiers problèmes d'impayés ou de conflits constatés, l'OPAC fait un signalement au maire qui a, dans un premier temps, toute liberté pour agir seul ou en associant éventuellement les services sociaux de sa commune. De nombreux maires, d'après les dires de notre interlocutrice, semblent exercer cette autorité avec une grande efficacité (la peur du qu'en dira-t-on jouant son rôle) pour le plus grand soulagement de l'OPAC.

Quels sont alors les intérêts réciproques des élus d'une part et de l'OPAC d'autre part à vouloir développer le logement social dans cette région rurale?

Du côté des maires, les motivations semblent liées à la gestion communale du développement. Il s'agit tout d'abord de maintenir voire de développer la population pour sauvegarder la présence des services et commerces. Dans cette lutte, les maires doivent accepter de prendre le risque d'accueillir des populations extérieures, qu'ils ne connaissent pas et qui parfois ont peu de liens locaux si ce n'est celui de la résidence. Il s'agit ensuite de gérer un patrimoine bâti communal parfois délabré et souvent trop coûteux pour les finances locales. À ce niveau, les communes sont très demandeuses car elles semblent gagnantes sur toute la ligne dans leur relation avec l'OPAC : en effet, en échange d'un bail emphytéotique ou d'une cession des bâtiments à l'organisme bailleur, c'est ce dernier qui assure le coût et la gestion de leur réhabilitation et de leur mise en location. Le développement du logement social peut ainsi servir l'objectif d'une réhabilitation de cœur de village (exemple de Mezille, où la réhabilitation a concerné une ancienne bâtisse sur la place de l'Église) ou la réaffectation des bâtiments liés à la présence de l'État, aujourd'hui remise en cause, vers une fonction résidentielle (exemple de Saint-Fargeau où l'ancienne gendarmerie a été transformée en cinq logements). Il s'agit aussi de répondre à la demande des administrés y compris des plus démunis, et notamment de maintenir sur place les jeunes couples. Ceux-ci, face à la concurrence foncière alimentée par l'arrivée des résidents secondaires et des retraités d'Île-de-France et à la faiblesse du parc locatif privé, n’ont souvent d'autres choix que de partir se loger ailleurs. Ce point, qui nous a été signalé à plusieurs reprises lors de nos entretiens avec les élus locaux, témoigne d'une prise de conscience des communes et d'un changement de contexte par rapport à celui décrit dans le rapport sur le logement locatif en milieu rural, publié en 1988 (Coulmin). L'auteur, à travers une comparaison entre catégories d'âge, statuts du logement occupé et types d'espace, en conclut que l'accès à la propriété est beaucoup plus précoce en milieu rural. Les facteurs explicatifs avancés sont liés à une accessibilité alors plus aisée à la propriété en milieu rural, mais aussi à la faiblesse de l'intervention publique dans le logement locatif, par rapport aux efforts fournis par les collectivités urbaines. C'est dire si depuis, le comportement des élus s'est 
ouvert sur l'enjeu de l'accès aux logements locatifs pendant que les conditions d'accès au marché immobilier et foncier se resserraient d'autant. Enfin, ce maintien sur place peut-être associé à la recherche d'une certaine mixité de population. L'OPAC s'oriente en effet, à la demande des élus, vers la réalisation de lotissements associant des logements d'insertion, des logements sociaux et des logements en accession à la propriété. Les élus de Puisaye-Forterre ne semblent pas isolés dans ce type de stratégie qui utilise le levier du logement locatif comme levier de développement. Sur le site internet de Mairie Conseil, des initiatives communales en matière de logement locatif pour impulser un développement démographique sont ainsi montrées en exemple.

Du côté de l'OPAC, l'implication en milieu rural semble s'inscrire dans une stratégie de gestion locative et de croissance de l'activité.

La gestion locative s'avère en milieu rural plus aisée du fait de l'appui direct des maires d'une part et de la situation de quasi-monopole exercé sur le parc locatif d'autre part. C'est en outre un marché en expansion, comme le montre la diminution du taux de vacance, quelle que soit la taille des communes. La demande croissante vient, nous l'avons vu, autant des ménages locaux qui n'ont pas accès à la propriété que des ménages urbains attirés par le cadre de vie. Le coût de la construction est incomparablement moins cher qu'en ville et les ressources foncières encore disponibles sont beaucoup plus importantes. En outre, les communes n’hésitent pas à passer des conventions avec l'OPAC en lui mettant à disposition du foncier pour la construction de nouveaux logements.

Ce contexte favorise une stratégie ambitieuse de développement de la construction qui passe par une politique d'acquisition du foncier et de diversification de l'offre.

Plusieurs instruments ont été mis en place pour satisfaire cet objectif de développement. L'OPAC a recruté depuis un an deux prospecteurs fonciers qui « traquent » toutes les opérations foncières et qui étudient toute proposition : de la maison à réhabiliter au terrain à construire. En un an, ces agents ont constitué un réseau de contacts comprenant aussi bien les agents immobiliers, les notaires, les propriétaires privés que les Safer, sans oublier les élus locaux. Une société, filiale de l'OPAC, Yonne Habitation a été créée pour développer le logement locatif en pavillon individuel permettant d'accueillir des familles dont les ressources excèdent de $30 \%$ les plafonds classiques. Ce bailleur social entend bien développer son offre et ainsi son activité en prenant appui sur le formidable réservoir immobilier et foncier de l'espace rural. Est-ce l'un des derniers avatars de l'étalement urbain? L'OPAC et les élus locaux ne participent-ils pas, à partir de stratégies différentes, à la périurbanisation? Pour éclairer ces questions, plaçons-nous à l'échelle du pays : l'histoire politique de sa genèse peut permettre de comprendre en effet le relatif émiettement communal qui alimente les stratégies individuelles des élus pour attirer et accueillir de la population.

\section{La Puisaye-Forterre : un pays rural sous influence urbaine}

La Puisaye-Forterre fait partie de ces régions classées dans le rural isolé, alors même qu'elle est sous forte influence urbaine. Du fait des deux autoroutes (A6, A77) qui bordent ce territoire, son accessibilité notamment depuis Paris et l'Île-de-France (IDF) est très forte (à $1 \mathrm{~h} 15$ de Paris depuis la sortie nord-est). Cette desserte favorise l'implantation de résidents secondaires (30\% en moyenne), de jeunes retraités et de cadres supérieurs travaillant en IDF. Autant d'éléments qui alimentent la pression foncière et immobilière. Auxerre, à une demi-heure à l'ouest du pays, offre également un débouché en termes de services et d'emplois. Malgré l'influence urbaine, la Puisaye-Forterre conserve un caractère rural prononcé. Avec une densité moyenne de 20 habitants au $\mathrm{km}^{2}$, elle parvient à tirer avantage de son attractivité résidentielle : le solde migratoire très positif entre les deux derniers recensements (+2 366 personnes) permet désormais de contrebalancer le solde naturel encore négatif (-1 715). La ruralité y prend donc un visage en recomposition. C'est sur ce terreau que s'est mise en place la démarche de pays dès 1996. Au départ n'existait pas la 
Puisaye-Forterre, mais seulement la Puisaye. Cette terre a été le fief d'un élu politique d'envergure, J.-P. Soisson, qui y a acquis les appuis nécessaires à son élection à la mairie d’Auxerre. Ce soutien, J.-P. Soisson, devenu député-maire, ne l'oublia pas et fit en retour, énormément pour la Puisaye (Abélès, 1989). Ce pays peu favorisé sur le plan agricole, vidé par l'exode, a été en effet alimenté par un soutien public et financier que J-P. Soisson mit beaucoup de zèle à obtenir. Le développement d'une culture de guichet a abouti à la structuration de quelques fiefs locaux entretenus par le clientélisme. L'investissement de Soisson dans cette région dépasse cependant le simple calcul stratégique. C'est lui qui en effet est allé convaincre les élus de Puisaye et de Forterre de s'allier dans un même projet de pays. La structuration en communautés de communes suit néanmoins étroitement les limites des cantons dont les conseillers constituent les relais de Soisson. Le leadership des conseillers généraux explique sans doute les difficultés à fonctionner à l'échelle du pays. La vision du pays des élus que nous avons interviewés est celle d'une fédération non achevée de communautés de communes, au sein desquelles il est difficile de dépasser les intérêts communaux pour penser et localiser les projets.

C'est sur ce terreau d'émiettement communal, soutenant une culture du développement alimenté de l'extérieur, que fleurissent les lotissements sociaux au fil des demandes des maires et au gré des priorités et des critères de l'OPAC.

\section{Un lotissement au milieu des champs}

V. est un village de Forterre, entouré de champs cultivés sur les riches terres que se partagent quelques grands exploitants. À gauche, un alignement de maisons de belle allure suit la route. Â droite, à trois kilomètres du village, treize pavillons jumelés, de plus modeste facture, se font face. C'est de ce côté-ci de la route que nous nous sommes arrêtée pour rencontrer trois des occupants de ce lotissement HLM. Préalablement interviewé, le maire, femme d'un riche exploitant, nous avait finalement confié que les habitants de ce lotissement - "ne posant pas problème et restant bien tranquillement entre eux »-avaient tout de même quelques difficultés à payer leur loyer et à s'insérer dans la vie du village. Ce point de départ nous a conduit auprès des différents protagonistes de cette histoire qui évoque le partage de la terre, la place des pauvres et les relations ville-campagne.

Les élus de $\mathrm{V}$. ont du faire face depuis plusieurs décennies à une baisse continue de la population. Il faut attendre 1990 pour que la courbe remonte grâce au solde migratoire, soit trois ans après que l'OPAC de l'Yonne ait accepté, à la demande du conseil municipal, d'y construire treize pavillons. Personne n'est de trop dans cette commune qui compte tout juste 660 habitants (1999) et dont le maire cherche à maintenir le statut de second bourg centre du canton. V. n'étant qu'à une demi-heure d'Auxerre, peut profiter de la demande croissante de logements de la part de ménages "qui n’hésitent pas à faire $27 \mathrm{~km}$ pour aller travailler à Auxerre " (maire). La commune a pu vendre ainsi pour « une somme dérisoire » une part de ses réserves foncières à l'OPAC.

Ce processus peut être alimenté, de l'extérieur, par les dynamiques centrifuges de ségrégation urbaine qui repoussent toujours plus loin dans les périphéries rurales les ménages modestes. Le comportement des élus locaux se distingue en tout cas des stratégies sélectives à l'œuvre dans les villes-centre et se trouve aux antipodes de celles qui se développent dans le périurbain proche sous les formes d'une « clubisation ${ }^{4} »$ de ces communes (Charmes, 2005). Est-ce un nouvel avatar de la domination des campagnes par la société englobante, dont le schéma a été mis en lumière par la sociologie rurale (Bodiguel, 1975)? Dans ce cadre explicatif, les élus ruraux n'ont d'autre choix pour maintenir la taille démographique de leur commune que de s'en remettre au bon vouloir des bailleurs sociaux urbains et de développer à l'échelle de leur commune les «fonctions d'accueil

4. La «clubisation » consiste en stratégie foncière développées par les élus municipaux visant à faire de leur commune un territoire exclusif, en s'opposant ou limitant la construction de logements sociaux et en imposant des dimensions parcellaires excluant les couches les plus modestes. 
pour populations spécifiques ». C'est ce que trahissent sans doute en partie les comportements asymétriques de nos interlocuteurs : l'embarras du maire à parler de ces « habitants là » et de son rôle de médiatrice, d'une part, et la facilité avec laquelle la responsable de l'OPAC a déployé les chiffres montrant l'importance de son implantation rurale, d'autre part. Mais cet embarras renvoie aussi à une véritable difficulté à situer le lieu du lotissement dans l'espace social communal, lieu que le maire a pourtant contribué à créer et qui lui échappe en partie à présent. Durant l'entretien mené avec l'élu, trois moments révèlent ce décalage entre le lieu du lotissement et l'espace communal.

Tout d'abord, le maire évoque de façon ambivalente le lotissement comme un espace sans problème mais marqué par l'entre-soi de ses habitants. Cet entre-soi situe le lotissement « hors de la commune ", c'est-à-dire de la visibilité et du contrôle social local. Le second moment est celui où le maire considère le lotissement comme un lieu faisant partie de la commune mais dont la gestion s'avère difficile du fait de l'extériorité de ses habitants. Extérieurs du point de vue des critères habituels d'appartenance car les habitants sont choisis en lien avec l'opérateur extérieur (OPAC) et extérieurs du point de vue du quotidien car ils vivent en grande partie à l'extérieur de la commune (travail, achats, loisirs). Enfin, alors que le magnétophone est arrêté, le maire, livrant une confidence, monte en généralité : «Vous savez, le problème c'est que maintenant les gens sont trop heureux. » Issue d'une famille de la paysannerie locale aisée, cette élue rappelle alors le rural de sa jeunesse, ruralité où l'existence de chacun était tracée dans les sillons de l'héritage et du labeur. On avait ce que l'on méritait et chacun était à sa place.

Ses occupants habitent la commune mais vivent en grande partie au dehors : ils travaillent à Auxerre ou dans des pôles d'emplois éloignés, font leurs courses dans les centres commerciaux périphériques. On se connaît mais jamais entièrement, jamais sous toutes les facettes que recouvrent aujourd'hui les différents espaces de vie séparés par la distance-temps (travail, loisirs, achats, associations). Rien finalement que de très banal si l'on compare la vie des autres résidents que nous avons interviewés dans d'autres communes du pays : l'interconnaissance des anciennes communautés villageoises a disparu, l'autochtonie et l'ancrage sont limités aux plus anciennes générations. Les agriculteurs ont suivi leur formation à l'extérieur et sont intégrés à des marchés nationaux et à des canaux d'information européens. Ici, comme dans le village métamorphosé de P. Dibie (2006, p. 33), «Pour la majorité des habitants, l'histoire individuelle ne se construit plus au village. »

Le lotissement social pavillonnaire dévoile alors sa nature : c'est un lieu condensé et révélateur. Il est révélateur des transformations du rural car il condense dans sa forme et son contenu des éléments ailleurs dilués et moins visibles : l'urbanisation des modes de vie et l'influence urbaine sur les modes de développement des communes rurales. Ce lieu, nouveau dans sa forme et son peuplement, agit par contraste avec l'environnement social et architectural où il prend corps. C'est peut-être pourquoi, dans les discours recueillis auprès du maire et des occupants du lotissement, ce lieu condensé d'urbanité semble vécu d'une manière assez proche de ce que M. Augé (1992) décrit à propos des « non-lieux » : non identitaire, non relationnel et non historique.

Sa forme, un lotissement isolé de la trame du village à l'habitat groupé, semble indiquer une rupture du bâti et du peuplement. Le village s'étend au-delà de sa structure initiale, par une annexe qui est autant la sienne que celui de la ville voisine participant à son peuplement. Ce peuplement s'effectue selon des normes en partie urbaines négociées par le maire avec le bailleur social. L'histoire est ici écrite de l'extérieur, aux termes de savants compromis entre différents opérateurs (élus, lotisseurs, bailleurs sociaux). La configuration du lotissement, des pavillons, répond à des questions de coût et de normes d'habitat. L'espace public est dédié à la circulation et au stationnement de l'automobile qui permet de s'échapper vers la ville.

C'est un lieu qui introduit également une rupture par rapport aux processus identitaires habituels. En effet, ses occupants doivent produire un dossier pour attester de leurs droits, ce qui leur permet ensuite d'être affranchis de toute autre identité que celle d'occupant d'un espace où l'anonymat est un gage de liberté individuelle. Espace de transit, il opère avant tout par la coexistence entre usagers engagés dans des cycles de vie et des trajectoires différentes. Ainsi, même les deux 
personnes qui ont acheté leur pavillon (programme d'accession à la propriété de l'OPAC) nous ont dit qu'elles ne finiraient pas leurs jours ici. Elles pensent repartir vers la ville d'Auxerre d'où elles sont venues et où elles ont gardé un pied-à-terre. Enfin, l'aspect relationnel semble ici atrophié. Du côté du maire, l'accent est mis sur la «non-participation » des occupants du lotissement à la vie de la commune : ils ne fréquentent pas les quelques commerces du village et on ne les voit guère aux activités de l'école. Du côté des occupants du lotissement, on souligne la relégation spatiale et sociale dans laquelle s'organise le quotidien : l'absence de trottoir et d'éclairage public pour se rendre sur les chemins de l'école au village, l'impossibilité financière de participer aux sorties scolaires, les commerces locaux dont les prix sont inabordables.

Si cet espace est en partie, et seulement en partie, non identitaire, non relationnel, non historique, il peut être considéré en partie comme un «non-lieu ». Pour le reste, et peut-être l'essentiel, il constitue un lieu en devenir, mêlant des éléments d'urbanité à une trame rurale recomposée socialement. Certains éléments en lisière de ce «non-lieu » peuvent ainsi en être les indicateurs furtifs. Tels ces deux propriétaires des maisons situées à l'entrée du lotissement qui sont devenus amis par les hasards de trajectoires parallèles qui les ont amenés à s'allier aux occupantes respectives de leurs logements actuels. À travers les relations des deux couples voisins se dessinent les contours d'un lieu de co-habitation véritable, fait d'échanges réciproques, de bavardages impromptus, d'invitations à tour de rôle. Sur une autre bordure, celle qui sépare le pavillon de la famille Havene des terres agricoles appartenant à une famille d'éleveurs avicoles, un autre quotidien s'invente empruntant à la ruse et au braconnage des règles d'usage des lieux (De Certeau, 1980). Les premiers se sont pris d'amitié pour leurs voisins agriculteurs. Les Havene leur achètent œufs et poulets, et les éleveurs leur louent un bout de terrain, tout ce commerce échappant bien sûr à l'officialité. Peut-être qu'ici comme ailleurs, l'ancrage de certains donnera-t-il prise à la genèse d'un lieu qui se vit, se construit, se raconte, collectivement et intimement?

En ce sens, le lotissement au milieu des champs doit être considéré comme un objet socio-spatial révélateur et complexe. Il contient du non-lieu mais aussi du lieu en devenir. Il révèle l'urbanité du mode de vie et des ressorts du développement rural.

\section{Conclusion}

Cette analyse se situe en quelque sorte à la lisière du champ d'étude sur le périurbain. Étudier cet objet à partir d'espaces ruraux et d'une forme particulière, le lotissement social, peut néanmoins contribuer à saisir ce qui se profile en devenir, sur les fronts pionniers de la périurbanisation. L'implantation de lotissements pavillonnaires dans l'espace villageois introduit une forme d'habitat radicalement urbaine remettant en question ouvertement l'interconnaissance et le contrôle local des relations. Pour autant, si elle porte en germe la fragmentation sociale, par l'anonymat des relations et la multi-localisation de ses habitants, cette forme urbaine se mâtine d'éléments proprement ruraux : ancrage de certains habitants, économie informelle, bricolage... La greffe urbaine en milieu rural, plus qu'un non-lieu, ressemble plutôt à un interstice (Hatzfeld, 1997) qui permet d'intégrer la nouveauté, l'informel, l'étranger que les espaces centraux (village-centre comme centre-ville) ne peuvent intégrer directement.

Dans les débats animant la réflexion sur l'étalement urbain, le périurbain est souvent pris comme une annexe résidentielle conquise par la ville sur un espace vierge. Nous avons cherché ici à éclairer ce point aveugle qui ignore les deux sens géographiques dans lesquels se développe la périurbanisation. Ainsi dans les transactions qui s'opèrent entre maires ruraux et le bailleur social urbain, il est question autant d'accueillir les ménages urbains en recherche d'espace que de loger les ruraux en désir d'ancrage. Ces ruraux vont pour partie trouver emplois et services en ville (il s'agit donc d'une périurbanité rurale) tandis que certains des urbains, une fois installés, cherchent à rapprocher leur emploi de leur résidence et s'y ancrent parfois pour leur retraite (il s'agirait d'une ruralisation d'une partie de la population urbaine). 
Le périurbain gagnerait ainsi à être saisi comme un processus qui se développe à travers des formes diverses selon les contextes où il prend corps et selon les types d'acteurs qu'il met en contact.

\section{Bibliographie}

ABÉLÈs (M.), 1989. - Jours tranquilles en 89 - Ethnologie politique d'un département, Paris, Odile Jacob, $352 \mathrm{p}$.

Alphandery (P.), Pinton (F.), 1998. - « Le Pays, territoire de l'environnement? La protection de la nature en Puisaye », Sciences de la Société, 45, p. 119-138.

Augé (M.), 1992. - Non-Lieux - Introduction à une anthropologie de la surmodernité, Paris, Seuil, 150 p.

Bodiguel (M.), 1975. - Les paysans face au progrès, Paris, Presses de la Fondation Nationale des Sciences Politiques, $177 \mathrm{p}$.

Charmes (E.), 2005. - Les petites communes périurbaines face aux nouvelles formes de l'entre-soi, Rapport intermédiaire pour le PUCA, Ministère de l'Equipement et des Transports, Paris, 53 p.

Coulmin (P.), 1988. - Le locatif en milieu rural, Rapport pour la Fédération nationale de l'habitat rural et de l'aménagement du territoire rural, Paris, $145 \mathrm{p}$.

De Certeau (M.), 1980. - L'invention du quotidien, tome 2, Habiter, cuisiner, Paris, Gallimard, coll. « Folio Essais », 416 p.

Delannoy (M.), 2005. - « L'habitat social en milieu rural dans le Nord-Pas-de-Calais, un enjeu social d'intégration et de socialisation », Colloque Faire Campagne, Rennes 17-18 mars, 15 p.

Dibie (P.), 2006. - Le village métamorphosé - Révolution dans la France profonde, Paris, Plon, Coll. Terre humaine, $405 \mathrm{p}$.

DonZELOT (J.), 2004. - « La ville à trois vitesses. Gentrification, relégation, périurbanisation », Esprit, n 3-4, mars-avril, p. 14-39.

Dunoyer De Segonzac (P.), 2004. - De la cité à la « résidence». Repères pour la résidentialisation, Union sociale pour l'habitat, 01/2004, $100 \mathrm{p}$.

HATZFELD (H.), 1997. - Interstices urbains et nouveaux types d'emplois, Rapport pour le programme Ville et emploi du Plan Urbain, Paris, Idacte-Interstices, 108 p.

Le Marec (A.), 2004. - Cherche toit désespérément, Pour, nº 182, p. 217-222.

Minodier (C.), 2004. - «Le parc locatif récent : davantage de maisons et de petits immeubles », Insee Première, $\mathrm{n}^{\circ}$ 957, $\mathrm{1}^{\mathrm{er}}$ avril 2004, p. 1.

Perrier-Cornet (P.) (dir), 2002. - Repenser les campagnes, Paris, Éditions de l'Aube/Datar, 279 p.

RÉMY (J.), 1998. - « La transaction sociale : forme de sociabilité et posture méthodologique », dans FrEYNET (M-F.) (coord.), Les transactions aux frontières du social, Lyon, Ed. Chronique sociale, p. 20-42.

VANONI (D.), 2007. - « La dimension territoriale de la crise du logement », Études foncières, n 128 , juilletaoût, p.7-13

Zamboni (C.), 2007.- Le logement à l'épreuve du développement rural. L'exemple de cœurs de villages, Mémoire de Master 2, SADL, Université Lyon 2, 69 p.

Cet article a été reçu le 3 mai 2007 et définitivement accepté le 8 janvier 2008. 